\title{
Utilização de Resíduos Urbanos e Agroflorestais para Produção de Mudas de Eucalyptus benthamii e Mimosa scabrella
}

\author{
Dagma Kratz ${ }^{1}$, Ivar Wendling², Antonio Carlos Nogueira ${ }^{1}$, \\ Paulo Vitor Dutra de Souza ${ }^{3}$
}

\author{
${ }^{1}$ Departamento de Ciências Florestais, Universidade Federal do Paraná - UFPR, Curitiba/PR, Brasil \\ ${ }^{2}$ Propagação de espécies florestais, Embrapa Florestas, Empresa Brasileira de Pesquisa Agropecuária - EMBRAPA \\ Colombo/PR, Brasil \\ ${ }^{3}$ Departamento de Horticultura e Silvicultura, Universidade Federal do Rio Grande do Sul - UFRGS, \\ Porto Alegre/RS, Brasil
}

\begin{abstract}
RESUMO
Objetivou-se analisar o crescimento de mudas de Eucalyptus benthamii e Mimosa scabrella produzidas em substratos à base de biossólido (BIO), casca de arroz carbonizada (CAC) e substrato comercial à base de casca de pinus semidecomposta (SC), e correlacionar as propriedades dos substratos com o crescimento das mudas. Para tanto, foram formulados seis substratos: SC; BIO/ CAC (1/1, v:v); BIO/CAC (4/6, v:v); BIO/CAC (3/7, v:v); BIO/CAC $(2 / 8, \mathrm{v}: \mathrm{v})$, e BIO/CAC (1/9, v:v). Foram avaliados os seguintes aspectos: a altura da parte aérea; o diâmetro de colo; a biomassa seca da parte aérea e radicial; a facilidade de retirada do tubete, e a agregação das raízes ao substrato. Os resultados mostraram que todos os substratos foram viáveis para Eucalyptus benthamii, enquanto que, para Mimosa scabrella, aqueles à base de biossólido não foram viáveis, devido ao teor de matéria orgânica, ao $\mathrm{pH}$, à condutividade elétrica e à salinidade.
\end{abstract}

Palavras-chave: eucalipto, bracatinga, biossólido.

\section{The Use of Municipal and Agroforestry Waste in the Production of Eucalyptus benthamii and Mimosa scabrella Seedlings}

\begin{abstract}
In this study, we aimed to analyze the growth of Eucalyptus benthamii and Mimosa scabrella seedlings produced on substrates based on biosolids (BIO), carbonized rice hulls (CRH) and semi-decomposed pine husk (SC), and to correlate the properties of the substrates to the growth of seedlings. To this end, six substrates were prepared: SC, BIO/CRH $(1 / 1, \mathrm{v}: \mathrm{v}), \mathrm{BIO} / \mathrm{CRH}$ (4/6, v:v), BIO/CRH (3/7, v:v), BIO/CRH (2/8, v:v) and BIO/CRH (1/9, v:v). The following variables were assessed: shoot height, stem diameter, shoot dry biomass, root dry biomass, ease of seedling removal from the cartridge, and substrate root aggregation. The results showed that all substrates were viable for Eucalyptus benthamii, whereas for Mimosa scabrella, subtracts based on biosolids were not viable because of organic matter, $\mathrm{pH}$, electrical conductivity, and salinity.
\end{abstract}

Keywords: Eucalyptus, bracatinga, biosolids. 


\section{INTRODUÇÃO}

Nas regiões mais frias, como o sul do Brasil, Eucalyptus benthamii Maiden et Cambage é uma das espécies mais importantes economicamente, visto apresentar boa aptidão, principalmente para a produção de madeira com fins energéticos e sólidos madeiráveis, associada à resistência a geadas (Paludzyszyn Filho et al., 2006).

No que se refere às espécies nativas, destaca-se a produção de Mimosa scabrella Benth (bracatinga), principalmente por pequenos produtores rurais da região de ocorrência da Floresta Ombrófila Mista, tendo como principal uso a madeira como lenha, uma vez que a sua rotação é bastante curta, entre seis e oito anos, e não se exigem muitos tratos silviculturais (Rotta \& Oliveira, 1981; Carpanezzi et al., 1997).

Para atender à necessidade de produção de mudas, muitos esforços têm sido realizados para melhorar a qualidade e reduzir os custos de produção; dentre os fatores que influenciam na qualidade, está o substrato, sendo este o meio em que as raízes se desenvolvem, fornecendo suporte estrutural às mudas e também as concentrações necessárias de água, oxigênio e nutrientes (Carneiro, 1995; Hartmann et al., 2011).

No mercado, podem ser encontrados substratos prontos para produção de mudas, sendo a casca de pinus semidecomposta e a vermiculita $(9: 1, \mathrm{v} / \mathrm{v})$ o principal produto comercializado (Caldeira et al., 2011). Entretanto, atualmente, destaca-se a baixa oferta de casca de pinus para fabricação de substratos, devido à competição com o mercado de energia e à diminuição de plantios de pinus no Brasil (ABRAF, 2012), bem como à necessidade premente do aumento no número de mudas a serem produzidas.

Dessa forma, devem-se aumentar os estudos em relação aos substratos, a fim de apresentar novas possibilidades de formulação desse produto, como a utilização de resíduos agroflorestais e urbanos para a produção de mudas; esta representa uma alternativa viável, pois grandes volumes desses produtos são gerados, expressando um problema ambiental, se não for apresentado um destino final adequado.

Dentre os resíduos que representam um grande passivo ambiental, está o biossólido, nome comercial do lodo de esgoto, o qual, após sofrer um processo de estabilização, constitui a parte sólida do esgoto (Assenheimer, 2009). Uma das utilizações compreende o fornecimento de matéria orgânica na composição de substratos para formação de mudas florestais, apresentando teores razoáveis de nutrientes, com destaque para nitrogênio e fósforo (Guerrini \& Trigueiro, 2004). A utilização desse elemento como componente para formulação de substratos apresentou resultados positivos em diversos trabalhos (Faustino et al., 2005; Morais et al., 1997; Guerrini \& Trigueiro, 2004; Cunha et al., 2006).

Quanto à casca de arroz carbonizada, esse componente de substrato vem sendo amplamente utilizado na produção de mudas, combinado com outros elementos, como fibra de coco, turfa e casca de pinus. É um produto extremamente leve, estéril, de fácil manuseio, alta porosidade, boa aeração e baixa capacidade de retenção de água (Wendling \& Gatto, 2002), além de ser estável física e quimicamente. Segundo Couto et al. (2003), a baixa densidade da casca de arroz carbonizada é uma característica importante quando se deseja aumentar a porosidade total do substrato, proporcionando maior drenagem da água de irrigação e uma melhor aeração do sistema radicial da muda.

Baseado no exposto, o presente trabalho objetivou analisar o crescimento de mudas de Eucalyptus benthamii e Mimosa scabrella produzidas em substratos renováveis à base de biossólido, casca de arroz carbonizada e casca de pinus semidecomposta, além de analisar a influência das propriedades dos substratos no crescimento das mudas.

\section{MATERIAL E MÉTODOS}

Os experimentos foram executados no Laboratório de Propagação de Espécies Florestais da Empresa Brasileira de Pesquisa Agropecuária - Embrapa Florestas, localizado em Colombo, Paraná, situado a $25^{\circ} 19^{\prime} 17^{\prime \prime}$ de latitude S e $49^{\circ} 09^{\prime} 39^{\prime \prime}$ de longitude W. O clima da região, de acordo com o Sistema Internacional de Köppen, é do tipo Cfb (clima subtropical úmido).

Em janeiro de 2010, realizou-se a semeadura de Eucalyptus benthamii em tubetes de $55 \mathrm{~cm}^{3}$. Após a semeadura, as bandejas foram acondicionadas em estufa de vidro (três irrigações diárias de $10 \mathrm{~min}$ 
com vazão de $144 \mathrm{~L} \mathrm{hora}^{-1}$ ), onde permaneceram por 60 dias, seguindo para área de rustificação (quatro irrigações diárias de $30 \mathrm{~min}$ com vazão de $97 \mathrm{~L} \mathrm{hora}^{-1}$ ), sendo expostas diretamente ao sol por 30 dias.

Para Mimosa scabrella, em março de 2010, efetuou-se a superação de dormência das sementes com imersão em água a $80{ }^{\circ} \mathrm{C}$ por 18 horas (Bianchetti, 1981). Foram semeadas manualmente duas sementes por tubete de $55 \mathrm{~cm}^{3}$, cobertas com uma fina camada $(0,5 \mathrm{~cm})$ de vermiculita. Após a semeadura, as bandejas foram acondicionadas em estufa de vidro por 120 dias, seguindo para área de rustificação por 30 dias.

Foi utilizado substrato comercial à base de casca de pinus semidecomposta (SC) e misturas de biossólido (BIO) e casca de arroz carbonizada (CAC) para a formulação de seis substratos, conforme as seguintes composições: S1: SC; S2: $50 \mathrm{BIO/} 50 \mathrm{CAC}$; S3: $40 \mathrm{BIO} / 60 \mathrm{CAC}$; S4: $30 \mathrm{BIO} / 70 \mathrm{CAC}$; S5: $20 \mathrm{BIO} / 80 \mathrm{CAC}$, e S6: $10 \mathrm{BIO} / 90 \mathrm{CAC}$, sendo as respectivas características físicas e químicas apresentadas na Tabela 1. A caracterização física e química dos substratos foi realizada no Laboratório de Substratos do Departamento de Horticultura e Silvicultura da Universidade Federal do Rio Grande do Sul, conforme a metodologia descrita na Instrução Normativa n. ${ }^{o} 17$ do Ministério da Agricultura, Pecuária e Abastecimento (Brasil, 2007) e Fermino (2003).

Os materiais foram misturados manualmente, juntos com a adubação de base $\left(1,5 \mathrm{~kg} \mathrm{~m}^{-3}\right.$ de substrato do fertilizante de liberação lenta de seis meses, da formulação 15: 10: 10, além de 3,5\% de Ca; 1,5\% de $\mathrm{Mg} ; 3,0 \%$ de S; 0,05\% de $\mathrm{Zn} ; 0,02 \%$ de B; 0,05\% de $\mathrm{Cu} ; 0,1 \%$ de $\mathrm{Mn} ; 0,5 \%$ de Fe, e 0,004\% de Mo).

Aos 30 dias, iniciou-se a adubação de crescimento [4 $\mathrm{g} \mathrm{L}^{-1}$ de Ureia, $3 \mathrm{~g} \mathrm{~L}^{-1}$ de superfosfato simples, 0,25 $\mathrm{g} \mathrm{L}^{-1}$ de FTE BR 10 (7\% Zn, 4\% Fe, 4\% Mn, $0,1 \%$ Mo, 2,5\% B, 0,8\% Cu) e $3 \mathrm{~g} \mathrm{~L}^{-1}$ de cloreto de potássio], realizada a cada sete dias até os 60 dias para Eucalyptus benthamii e até os 120 dias para Mimosa scabrella.

Quando as mudas foram transferidas para a área de pleno sol, 60 dias para Eucalyptus benthamii e 120 dias para Mimosa scabrella, iniciou-se uma adubação de rustificação (4 $\mathrm{g} \mathrm{L}^{-1}$ de sulfato de amônio, $10 \mathrm{~g} \mathrm{~L}^{-1}$ de superfosfato simples, $4 \mathrm{~g} \mathrm{~L}^{-1} \mathrm{de}$ cloreto de potássio, $1 \mathrm{~g} \mathrm{~L}^{-1}$ de FTE BR 10), realizada também a cada sete dias até o final do experimento.

Para avaliação da qualidade das mudas, foram medidos: a altura da parte aérea $(\mathrm{H})$; o diâmetro de colo (DC); a biomassa seca aérea (BSA); a biomassa seca radicular (BSR), e os índices 'facilidade de retirada de tubete (FRT)' e 'agregação das raízes ao substrato (AG)'. Para as avaliações de FRT e AG, foi utilizada a metodologia descrita em Wendling et al. (2007); esse método consiste em atribuir notas de zero a dez às variáveis, sendo zero a dificuldade máxima e dez a facilidade máxima de retirada das mudas, após três batidas na parte superior (boca) do tubete. Quanto à agregação das raízes ao substrato, as mudas sem os tubetes foram soltas em queda livre a

Tabela 1. Densidade aparente (Dap), Porosidade total (Pt), Macroporosidade (Macro), Microporosidade (Micro), Matéria Orgânica $(\mathrm{MO})$, potencial hidrogeniônico $(\mathrm{pH})$, Condutividade Elétrica $(\mathrm{CE})$, Capacidade De Troca Catiônica (CTC) e Teor Total de Sais Solúveis (TTSS).

Table 1. Bulk density (Dap), total porosity (Pt), macroporosity (Macro), microporosity (Micro), Organic Matter (MO), Potential Hydrogen ( $\mathrm{pH})$, Electrical Conductivity (CE) and total content of soluble salts (TTSS).

\begin{tabular}{|c|c|c|c|c|c|c|c|c|}
\hline \multirow{2}{*}{ Substratos } & Da & $\mathbf{P t}$ & Macro & Micro & MO & \multirow{2}{*}{$\begin{array}{c}\mathbf{p H} \\
\left(\mathbf{H}_{2} \mathbf{O}\right)\end{array}$} & \multirow{2}{*}{$\begin{array}{c}\mathrm{CE} \\
\mathrm{mS} \mathrm{cm}^{-1}\end{array}$} & \multirow{2}{*}{$\begin{array}{l}\text { TTSS } \\
\mathrm{g} \mathrm{L}^{-1}\end{array}$} \\
\hline & $\mathrm{kg} \mathrm{m}^{3}$ & \multicolumn{4}{|c|}{$\%$} & & & \\
\hline SC & 398,00 & 79,00 & 19,00 & 60,00 & 48,55 & 5,47 & 1,53 & 5,86 \\
\hline $\mathrm{BIO} / \mathrm{CAC}(1 / 1, \mathrm{v}: \mathrm{v})$ & 389,00 & 82,00 & 36,00 & 46,00 & 62,81 & 8,75 & 2,74 & 8,78 \\
\hline $\mathrm{BIO} / \mathrm{CAC}(4 / 6, \mathrm{v}: \mathrm{v})$ & 362,00 & 84,00 & 40,00 & 44,00 & 61,80 & 8,88 & 2,34 & 6,90 \\
\hline $\mathrm{BIO} / \mathrm{CAC}(3 / 7, \mathrm{v}: \mathrm{v})$ & 289,00 & 82,00 & 50,00 & 32,00 & 54,08 & 8,79 & 1,67 & 4,34 \\
\hline $\mathrm{BIO} / \mathrm{CAC}(2 / 8, \mathrm{v}: \mathrm{v})$ & 229,00 & 86,00 & 56,00 & 30,00 & 51,70 & 9,06 & 1,25 & 2,67 \\
\hline $\mathrm{BIO} / \mathrm{CAC}(1 / 9, \mathrm{v}: \mathrm{v})$ & 184,00 & 90,00 & 65,00 & 25,00 & 43,18 & 9,13 & 0,85 & 1,48 \\
\hline
\end{tabular}

SC: Substrato florestal comercial à base de casca de pinus semidecomposta; BIO: Biossólido; CAC: Casca de Arroz Carbonizada. 
um metro do solo e, ao torrão, foi atribuída uma nota de zero a dez, sendo zero para a muda totalmente esboroada e dez para o torrão íntegro (Figura 1).

$\mathrm{O}$ delineamento experimental utilizado foi o inteiramente casualizado, com cinco repetições de 20 plantas (unidade amostral) e seis tratamentos, para ambos os experimentos. Os dados foram submetidos ao Teste de Bartlett $(\mathrm{p}<0,05)$, a fim de verificar a condição de homogeneidade de variância e, em seguida, à Análise de Variância (ANOVA) $(\mathrm{p}<0,01$ e $\mathrm{p}<0,05)$, prosseguindo para o Teste de Scott-Knott $(p<0,01$ e $p<0,05)$, a fim de observar as diferenças entre as médias. Também foi realizada a Análise de Correlação de Pearson entre as variáveis biométricas das espécies e as características físicas e químicas dos substratos.

\section{RESULTADOS E DISCUSSÃO}

\subsection{Produção de mudas}

De maneira geral, verificaram-se diferentes respostas para as duas espécies estudadas, comprovando a necessidade de se avaliar o substrato antes de utilizá-lo em escala comercial. Para Eucalyptus benthamii, todos os substratos renováveis foram viáveis tecnicamente, enquanto que, para Mimosa scabrella, o biossólido apresentou-se inviável, em razão do baixo crescimento alcançado com a adição desse elemento na composição dos substratos (Tabela 2).
Para a altura de Eucalyptus benthamii, verificou-se que todos os substratos renováveis utilizados foram aptos para a produção de mudas, pois as mesmas apresentaram altura superior a $15 \mathrm{~cm}$, valor mínimo recomendado para o plantio em campo, segundo Wendling e Dutra (2010). Já para Mimosa scabrella, apenas com a utilização de SC as mudas apresentaram altura adequada para o plantio $(15 \mathrm{~cm})$ (EMBRAPA, 1988).

Quanto ao diâmetro do colo, as mudas de Eucalyptus benthamii não apresentaram diâmetro mínimo recomendado para o plantio, o qual, segundo Wendling \& Dutra (2010), é de $2 \mathrm{~mm}$. No entanto, Eucalyptus benthamii é uma espécie que apresenta reconhecidamente mudas de diâmetros menores do que a grande maioria das espécies de Eucalyptus. Note-se que os valores encontrados estão próximos aos observados em outras pesquisas. Trigueiro \& Guerrini (2003) verificaram diâmetro de colo médio de 1,85 mm aos 90 dias em mudas de Eucalyptus grandis produzidas em substrato comercial à base de casca de pinus semidecomposta. Bonnet (2001) observou, aos 106 dias, diâmetro médio de 1,51 mm em mudas de Eucalyptus viminalis produzidas em substrato contendo $70 \%$ de substrato comercial combinado com 30\% de biossólido compostado com resíduo verde, e Freitas et al. (2005) observaram diâmetro de 2,0 mm em mudas de Eucalyptus grandis e 1,80 $\mathrm{mm}$ em Eucalyptus saligna, produzidas em substrato à base de casca de arroz carbonizada e casca de eucalipto (50/50). Cabe ressaltar que, com ajustes na nutrição, provavelmente os valores de diâmetro poderão ser incrementados.
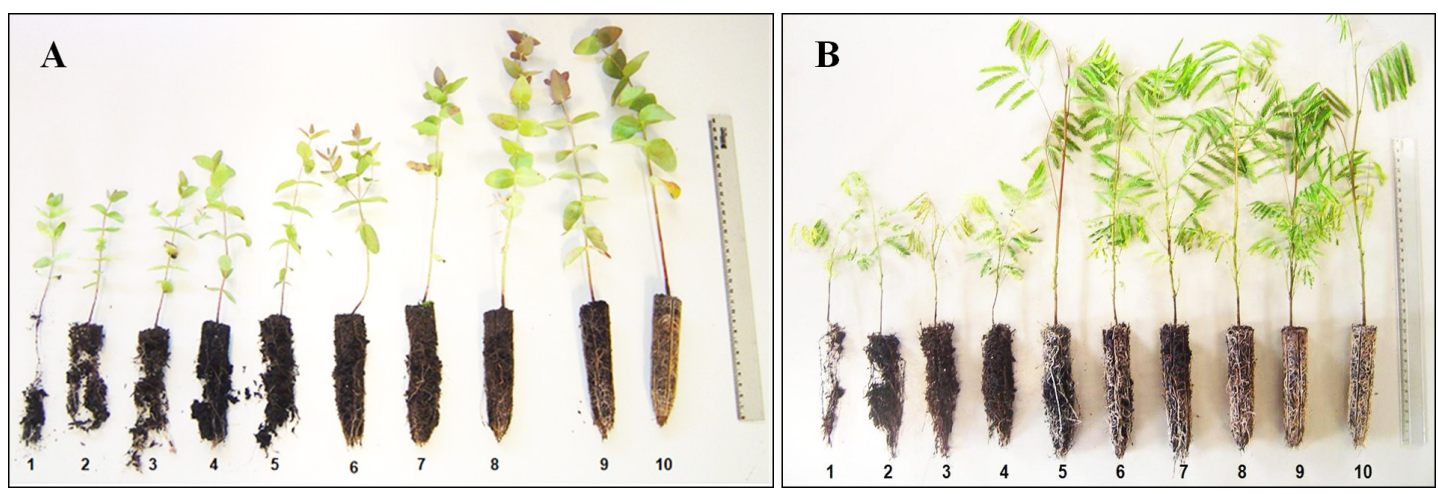

Figura 1. Índices de agregação das raízes ao substrato em mudas de Eucalyptus benthamii (A) e Mimosa scabrella (B). Figure 1. Index of aggregation of the substrate roots of Eucalyptus benthamii (A) and Mimosa scabrella (B). 
Para Mimosa scabrella, apenas com o SC, apresentou-se um diâmetro adequado para o plantio (maior do que $2 \mathrm{~mm}$ ). Observou-se que o aumento da proporção de $\mathrm{BIO}$ no substrato acarretou em decréscimo no diâmetro, denotando que esse elemento não foi viável tecnicamente nas proporções estudadas (Tabela 2).

Em relação à biomassa seca aérea e radicial, todos os tratamentos apresentaram comportamento semelhante, indicando que os substratos renováveis testados são viáveis tecnicamente para produção de mudas de Eucalyptus benthamii. Já para Mimosa scabrella, observou-se comportamento semelhante às demais variáveis analisadas, em que o aumento da proporção de $\mathrm{BIO}$ ocasionou diminuição do acúmulo de biomassa (Tabela 2), o que denotou a inviabilidade da utilização de BIO como componente de substratos para produção de mudas de Mimosa scabrella.

Segundo Gomes et al. (2002), a biomassa seca é um fator de grande importância no momento do plantio, visto que mudas com maior biomassa apresentam maior resistência às condições adversas do campo, promovendo maior sobrevivência e evitando gastos com replantio. A biomassa radicial, segundo Novaes (1998), sob o ponto de vista fisiológico, está diretamente ligada à sobrevivência e ao crescimento inicial em campo, em virtude da sua função de absorção de água e nutrientes.

A facilidade de retirada do tubete das mudas de Eucalyptus benthamii foi de média a alta. Já para
Mimosa scabrella, os substratos que apresentaram maior facilidade foram aqueles que proporcionaram o menor crescimento das mudas. A diferente resposta obtida pelas espécies está relacionada ao volume de raízes presente no substrato, em que a massa das raízes da Mimosa scabrella (0,381 g) é muito superior à massa de Eucalyptus benthamii $(0,128 \mathrm{~g})$, sendo que ambas as espécies foram produzidas em tubetes de $55 \mathrm{~cm}^{3}$.

Em se tratando da agregação das raízes ao substrato, para Eucalyptus benthamii, verificaram-se valores médios, enquanto que, para Mimosa scabrella, o SC apresentou alta agregação, sendo observado claramente que os substratos que proporcionaram maior crescimento foram os que tiveram maior qualidade do torrão formado. Esses resultados são similares aos encontrados por Kratz \& Wendling (2013) em mudas de Eucalyptus dunnii. Segundo esses autores, a baixa biomassa radicial e a falta de estrutura da casca de arroz carbonizada promovem a formação de um torrão com baixa agregação, dificultando o processo de expedição de mudas.

Para Wendling \& Delgado (2008), o substrato para produzir mudas em tubetes deve ser agregado o suficiente para que o torrão em volta da muda não se rompa quando a embalagem for retirada para plantio ou transporte, ocasionando exposição das raízes ao ressecamento e dificultando a pega e a sobrevivência das mudas. No entanto, se o substrato for muito coeso, haverá dificuldade em sua retirada da

Tabela 2. Altura (H), Diâmetro de Colo (DC), Biomassa Seca Aérea (BSA), Biomassa Seca Radicial (BSR), Facilidade de Retirada do Tubete (FRT) e Agregação das Raízes ao Substrato (AG) de mudas de Eucalyptus benthamii e Mimosa scabrella, produzidas em diferentes substratos aos 90 e 150 dias, respectivamente.

Table 2. Height (H), diameter (DC), aerial biomass (BSA), dry biomass radicial(BSR), ease of removal of seedlings of the cartridge (FRT) and the substrate root aggregation (AG) of Eucalyptus benthamii and Mimosa scabrella seedlings produced on different substrates 90 and 150 days, respectively.

\begin{tabular}{|c|c|c|c|c|c|c|c|c|c|c|c|c|}
\hline \multirow{3}{*}{ Substrato } & \multicolumn{6}{|c|}{ Eucalyptus benthamii } & \multicolumn{6}{|c|}{ Mimosa scabrella } \\
\hline & \multirow{2}{*}{$\begin{array}{c}\mathrm{H} \\
\mathrm{cm}^{-1}\end{array}$} & \multirow{2}{*}{$\begin{array}{c}\text { DC } \\
\mathbf{m m}^{-1}\end{array}$} & BSA & BSR & \multirow[t]{2}{*}{ FRT } & \multirow[t]{2}{*}{ AG } & $\mathbf{H}$ & DC & BSA & BSR & \multirow[t]{2}{*}{ FRT } & \multirow[t]{2}{*}{ AG } \\
\hline & & & \multicolumn{2}{|c|}{$\mathrm{mg}^{-1}$} & & & $\mathrm{~cm}^{-1}$ & $\mathrm{~mm}^{-1}$ & \multicolumn{2}{|c|}{$\mathrm{mg}^{-1}$} & & \\
\hline SC & $18,9 \mathrm{a}$ & $1,6 \mathrm{a}$ & $0,39 \mathrm{a}$ & $0,13 \mathrm{a}$ & $8,0 \mathrm{a}$ & $5,4 \mathrm{~b}$ & $21,2 \mathrm{a}$ & $2,5 \mathrm{a}$ & $0,83 \mathrm{a}$ & $0,38 \mathrm{a}$ & $3,8 \mathrm{~b}$ & $8,1 \mathrm{a}$ \\
\hline $\mathrm{BIO} / \mathrm{CAC}(1 / 1, \mathrm{v}: \mathrm{v})$ & $20,0 \mathrm{a}$ & $1,6 \mathrm{a}$ & $0,46 \mathrm{a}$ & $0,15 \mathrm{a}$ & $8,5 \mathrm{a}$ & $7,5 \mathrm{a}$ & $4,8 \mathrm{~d}$ & $0,9 \mathrm{~d}$ & $0,10 \mathrm{~d}$ & $0,07 \mathrm{~d}$ & $9,7 \mathrm{a}$ & $1,0 \mathrm{~d}$ \\
\hline $\mathrm{BIO} / \mathrm{CAC}(4 / 6, \mathrm{v}: \mathrm{v})$ & $20,2 \mathrm{a}$ & $1,8 \mathrm{a}$ & $0,39 \mathrm{a}$ & $0,12 \mathrm{a}$ & 7,9 a & $5,3 \mathrm{~b}$ & $9,1 \mathrm{c}$ & $1,2 \mathrm{c}$ & $0,24 \mathrm{~cd}$ & $0,19 \mathrm{~cd}$ & $9,3 \mathrm{a}$ & $2,0 \mathrm{~cd}$ \\
\hline $\mathrm{BIO} / \mathrm{CAC}(3 / 7, \mathrm{v}: \mathrm{v})$ & $21,9 \mathrm{a}$ & $1,9 \mathrm{a}$ & $0,53 \mathrm{a}$ & $0,15 \mathrm{a}$ & $8,5 \mathrm{a}$ & $6,2 \mathrm{ab}$ & $9,5 c$ & $1,4 \mathrm{bc}$ & $0,27 \mathrm{c}$ & $0,21 \mathrm{bcd}$ & $8,1 \mathrm{a}$ & $3,2 \mathrm{bc}$ \\
\hline $\mathrm{BIO} / \mathrm{CAC}(2 / 8, \mathrm{v}: \mathrm{v})$ & $18,9 \mathrm{a}$ & $1,6 \mathrm{a}$ & $0,39 \mathrm{a}$ & $0,12 \mathrm{a}$ & $7,2 \mathrm{a}$ & $5,3 \mathrm{~b}$ & $9,8 \mathrm{bc}$ & $1,5 \mathrm{bc}$ & $0,37 c$ & $0,25 \mathrm{abc}$ & $8,1 \mathrm{a}$ & $3,2 \mathrm{bc}$ \\
\hline $\mathrm{BIO} / \mathrm{CAC}(1 / 9, \mathrm{v}: \mathrm{v})$ & $20,3 \mathrm{a}$ & $1,8 \mathrm{a}$ & $0,44 \mathrm{a}$ & $0,14 \mathrm{a}$ & $8,1 \mathrm{a}$ & $6,5 \mathrm{ab}$ & $12,2 \mathrm{~b}$ & $1,6 \mathrm{~b}$ & $0,54 \mathrm{~b}$ & $0,34 \mathrm{ab}$ & $6,2 \mathrm{ab}$ & $4,2 \mathrm{~b}$ \\
\hline
\end{tabular}

Médias seguidas de uma mesma letra não diferem entre si, pelo teste de Tukey a 5\% de probabilidade de erro. SC - substrato comercial a base de casca de pinus e vermiculita; BIO - biossólido; CAC - Casca de Arroz Carbonizada. 
embalagem, podendo romper as raízes ou provocar danos no crescimento radicial das mudas.

\subsection{Influência das propriedades físicas e químicas no crescimento das mudas}

De maneirageral, verificou-se que as propriedades físicas dos substratos não apresentaram influência significativa no crescimento das mudas de ambas as espécies, enquanto que as propriedades químicas causaram um efeito significativo, principalmente para Mimosa scabrella (Tabela 3).

Ao se referir às propriedades físicas, verificou-se baixa variação nos valores de todas as propriedades. Já as propriedades químicas apresentaram diferenças mais expressivas, sendo tais fatores preponderantes, influenciando significativamente no crescimento das mudas de Mimosa scabrella.

$\mathrm{O}$ pH do SC $(5,47)$ foi considerado adequado, conforme a classificação de Kämpf (2005) (5,2 e 5,5) e Bailey et al. (2000) $(4,4$ a 6,2). Segundo Valeri \& Corradini (2000), em substratos com pH abaixo de 5,0 , pode ocorrer deficiência de nitrogênio, potássio, cálcio, magnésio e boro, enquanto que, em $\mathrm{pH}$ acima de 6,5, são esperadas deficiências de fósforo, ferro, manganês, zinco e cobre. Contudo, para Eucalyptus benthamii, as mudas apresentaram crescimento satisfatório, mesmo com $\mathrm{pH}$ acima da faixa considerada adequada, diferentemente das mudas de Mimosa scabrella (Tabelas 2 e 3), indicando que o eucalipto é menos exigente quanto ao $\mathrm{pH}$, ou seja, é mais tolerante às variações desse índice (Tabela 1). Tal característica também é verificada em outros trabalhos (Oliveira Junior et al., 2011; Kratz \& Wendling, 2013).

A matéria orgânica, conforme observado na Tabela 3, apresentou correlação negativa no crescimento das mudas de Mimosa scabrella. Porém, deve-se observar que o SC apresentou teores de matéria orgânica similares aos substratos à base de biossólido; logo, não se pode afirmar que apenas os altos teores de matéria orgânica foram prejudiciais ao crescimento das mudas de Mimosa scabrella. A resposta apresentada pelas mudas de Mimosa scabrella ao teor de matéria orgânica associada ao alto $\mathrm{pH}$ pode estar relacionada com a ecologia da espécie, visto que os bracatingais, segundo Carvalho (1994), ocorrem em solos pobres, ácidos ( $\mathrm{pH}$ variando entre 3,5 e 5,5) e bem drenados. Assim, conforme pode ser observado na Tabela 1, o pH do SC foi de 5,47, propiciando bom crescimento das mudas mesmo com altos teores de matéria orgânica, enquanto que o $\mathrm{pH}$ dos substratos à base de $\mathrm{BIO}$ variou de $8,75 \mathrm{a}$ 9,13, associado a altos níveis de matéria orgânica.

Tabela 3. Correlações entre as propriedades físicas e químicas dos substratos e as variáveis biométricas das mudas de Eucalyptus benthamii e Mimosa scabrella. Altura (H), Diâmetro do Colo (DC), Biomassa Seca Aérea (BSA), Biomassa Seca Radicial (BSR), Densidade aparente (Dap), Porosidade total (Pt), Macroporosidade (Macro), Microporosidade (Micro), Matéria Orgânica (MO), Potencial Hidrogeniônico (pH), Condutividade Elétrica (CE) e Teor Total de Sais Solúveis (TTSS).

Table 3. Correlations between the physical and chemical properties of substrates and biometric variables of seedlings of Eucalyptus benthamii and Mimosa scabrella. Height (H), Stem Diameter (DC), Aerial Dry Biomass (BSA), Radicial Dry Biomass (BSR), Aparent Density (Da), Total Porosity (PT), Macroporosity (Macro) and Microporosity (Micro), Organic Matter (MO), Potential Hydrogen $(\mathrm{pH})$, Electrical Conductivity $(\mathrm{CE})$ and Total Content of Soluble Salts (TTSS).

\begin{tabular}{|c|c|c|c|c|c|c|c|c|c|}
\hline & \multicolumn{4}{|c|}{ Eucalyptus benthamii } & \multicolumn{5}{|c|}{ Mimosa scabrella } \\
\hline & $\mathbf{H}$ & DC & BSA & BSR & & $\mathbf{H}$ & DC & BSA & BSR \\
\hline $\mathrm{Da}$ & $0,24^{\mathrm{ns}}$ & $-0,29^{\text {ns }}$ & $0,18^{\text {ns }}$ & $0,02^{\text {ns }}$ & $\mathrm{Da}$ & $-0,08^{\text {ns }}$ & $-0,06^{\text {ns }}$ & $-0,06^{\mathrm{ns}}$ & $-0,33^{\text {ns }}$ \\
\hline $\mathrm{Pt}$ & $-0,14^{\mathrm{ns}}$ & $0,21^{\mathrm{ns}}$ & $-0,30^{\mathrm{ns}}$ & $-0,28^{\mathrm{ns}}$ & $\mathrm{Pt}$ & $-0,10^{\mathrm{ns}}$ & $-0,13^{\mathrm{ns}}$ & $-0,10^{\mathrm{ns}}$ & $0,15^{\text {ns }}$ \\
\hline Macro & $0,04^{\text {ns }}$ & $0,40^{*}$ & $0,02^{\text {ns }}$ & $0,11^{\mathrm{ns}}$ & Macro & $-0,26^{\mathrm{ns}}$ & $-0,27^{\mathrm{ns}}$ & $-0,27^{\text {ns }}$ & $0,01^{\mathrm{ns}}$ \\
\hline Micro & $-0,09^{\text {ns }}$ & $-0,44^{*}$ & $-0,11^{\mathrm{ns}}$ & $-0,23^{\text {ns }}$ & Micro & $0,30^{\text {ns }}$ & $0,30^{\mathrm{ns}}$ & $0,31^{\mathrm{ns}}$ & $0,03^{\text {ns }}$ \\
\hline MO & $0,56^{*}$ & $-0,07^{\mathrm{ns}}$ & $0,41^{\star}$ & $0,19^{\text {ns }}$ & $\mathrm{MO}$ & $-0,77^{\star *}$ & $-0,74^{* *}$ & $-0,78^{\star *}$ & $-0,88^{\star *}$ \\
\hline $\mathrm{pH}$ & $0,40^{*}$ & $0,36^{\mathrm{ns}}$ & $0,27^{\text {ns }}$ & $0,22^{\text {ns }}$ & $\mathrm{pH}$ & $-0,75^{\star *}$ & $-0,75^{\star *}$ & $-0,76^{\star *}$ & $-0,54^{*}$ \\
\hline $\mathrm{CE}$ & $0,62^{\star *}$ & $-0,13^{\text {ns }}$ & $0,46^{\star}$ & $0,18^{\text {ns }}$ & $\mathrm{CE}$ & $-0,67^{\star *}$ & $-0,66^{\star *}$ & $-0,65^{\star *}$ & $-0,82^{\star *}$ \\
\hline TTSS & $0,50^{*}$ & $-0,25^{\mathrm{ns}}$ & $0,36^{\mathrm{ns}}$ & $0,09^{\text {ns }}$ & TTSS & $-0,43^{*}$ & $-0,42^{*}$ & $-0,40^{*}$ & $-0,64^{* *}$ \\
\hline
\end{tabular}

$\left.\left({ }^{* *}\right){ }^{*}\right)$ significativos nos níveis de $1 \%$ e $5 \%$ de probabilidade de erro, respectivamente; ${ }^{\left({ }^{n s}\right.}$ ) não significativo, pelo teste $\mathrm{F}$. 
Ao se referir à salinidade, segundo a classificação de Röber \& Schaller (1985), SC e BIO/CAC $(4 / 6, v: v)$ apresentaram salinidade extremamente alta $\left(5,0\right.$ a $\left.7,0 \mathrm{~g} \mathrm{~L}^{-1}\right)$; o $\mathrm{BIO} / \mathrm{CAC}(1 / 1, \mathrm{v}: \mathrm{v})$ foi considerado tóxico $\left(>7,0 \mathrm{~g} \mathrm{~L}^{-1}\right)$ e o $\mathrm{BIO} / \mathrm{CAC}$ (3/7, v:v), com salinidade alta $\left(2,0\right.$ a $\left.4,0 \mathrm{~g} \mathrm{~L}^{-1}\right)$ (Tabela 1). No entanto, os altos níveis de salinidade não prejudicaram o crescimento das mudas de Eucalyptus benthamii, demonstrando que essa espécie suporta concentrações mais elevadas de salinidade. Resultados similares foram observados em outras espécies do gênero Eucalyptus por Mendonça et al. (2010), os quais verificaram que mudas de Eucalyptus camaldulensis, Eucalyptus tereticornis e Eucalyptus robusta apresentaram-se resistentes à salinidade até a condutividade elétrica de $8,33 \mathrm{dS} \mathrm{m}^{-1}$, observando que a mesma não provocou redução no teor de clorofila, o que, em plantas sensíveis à salinidade, causa degradação desse elemento.

Para Mimosa scabrella, a salinidade influenciou negativamente o crescimento das mudas, exceto o SC, apresentando correlação negativa com as variáveis biométricas (Tabela 3). O menor crescimento das mudas de Mimosa scabrella nos substratos com alta condutividade elétrica e salinidade está relacionado ao fato de que altos valores para essa característica podem danificar as raízes e os pêlos radiciais, impedindo a absorção de água e nutrientes. Dessa forma, a atividade fisiológica é afetada e, consequentemente, diminui-se o crescimento das plantas (Rodrigues, 2002).

Os resultados verificados no presente trabalho comprovam a necessidade de se avaliar o substrato antes de utilizá-lo em escala comercial, visto que, para Eucalyptus benthamii, todos os componentes renováveis foram viáveis tecnicamente, enquanto que, para a Mimosa scabrella, o BIO apresentou-se inviável, em razão do baixo crescimento alcançado com a adição desse elemento para a composição dos substratos.

\section{CONCLUSÕES}

Os materiais renováveis avaliados para formulação de substratos - biossólido, casca de arroz carbonizada e casca de pinus semidecomposta - foram viáveis tecnicamente para a produção de mudas de Eucalyptus benthamii. Já para Mimosa scabrella, apenas o substrato à base de casca de pinus semidecomposta apresentou-se adequado.
As propriedades físicas e químicas dos diferentes substratos estudados foram adequadas para a produção de mudas de Eucalyptus benthamii, enquanto que, para a Mimosa scabrella, a densidade, a matéria orgânica, o $\mathrm{pH}$, a condutividade elétrica e a salinidade influenciaram negativamente no crescimento das mudas.

\section{STATUS DA SUBMISSÃO}

Recebido: 24/03/2013

Aceito: $26 / 08 / 2013$

Publicado: 31/12/2013

\section{AUTOR(ES) PARA CORRESPONDÊNCIA}

\section{Dagma Kratz}

Departamento de Ciências Florestais,

Universidade Federal do Paraná - UFPR,

CEP 80210-170, Curitiba, PR, Brasil

e-mail:dagkratz@yahoo.com.br

\section{REFERÊNCIAS}

Assenheimer A. Benefícios do uso de biossólidos como substratos na produção de mudas de espécies florestais. Revista Ambiência 2009; 5(2): 321-330.

Associação Brasileira de Produtores de Florestas Plantadas - ABRAF. Anuário estatístico da ABRAF 2012: ano base 2011. Brasília: ABRAF; 2012.

Bailey DA, Fonteno WC, Nelson PV. Greenhouse substrates and fertilization. Raleigh: North Caroline State University; 2000. Available from: http://www.cesncsu. edu/depts/hort/floriculture/plugs/ghsubfert.pdf.

Bianchetti A. Comparação de tratamentos para superar a impermeabilidade do tegumento de sementes de bracatinga (Mimosa scabrella Benth.). Boletim de Pesquisa Florestal 1981; 2: 57-68.

Bonnet BRP. Produção de mudas de Eucalyptus viminalis Lambill. (Myrtaceae), Schinus terebinthifolius Raddi (Anacardiaceae) e Mimosa scabrella Benth. (Mimosaceae) em substrato com lodo de esgoto anaeróbio digerido alcalinizado e compostado [dissertação]. Curitiba: Ciências Florestais, Universidade Federal do Paraná; 2001.

Brasil. Ministério da Agricultura, Pecuária e Abastecimento - MAPA. Instrução Normativa SDA no 17. Métodos Analíticos Oficiais para Análise de Substratos para Plantas e Condicionadores de Solo. Diário Oficial da República Federativa do Brasil, Brasília, DF, (2007 maio 24); Sec. 1(99).

Caldeira MVW, Wendling I, Penchel RM, Gonçalves EO, Kratz D, Trazzi PA. Propriedades de substratos 
para produção de mudas florestais. In: Caldeira MVW, Garcia GO, Gonçalves EO, Arantes MDC, Fiedler NC. Contexto e Perspectivas da Área Florestal no Brasil. Alegre: Suprema; 2011. p. 141-160.

Carneiro JGA. Produção e Controle de Qualidade de Mudas Florestais. Curitiba: UFPR/FUPEF; 1995. 451 p.

Carpanezzi AA, Pagano SN, Baggio AJ. Banco de sementes de bracatinga em povoamentos do sistema agroflorestal tradicional de cultivo. Boletim de Pesquisa Florestal 1997; 35: 3-19.

Carvalho PER. Mimosa scabrella Benth: bracatinga. In: Carvalho PER. Espécies florestais brasileiras: recomendações silviculturais, potencialidades e uso da madeira. Colombo: EMBRAPA-CNPF; 1994. p. 337-343. PMid:7660034.

Couto M, Wagner A Jr, Quezada AC. Efeito de diferentes substratos durante a aclimatização de plantas micropropagadas do porta-enxerto mirabolano 29c (Prunus cerasifera ehrh.) em casa de vegetação. Revista Brasileira de Agrociência 2003; 9(2): 125-128.

Cunha AM, Cunha GM, Sarmento RA, Cunha GM, Amaral JFT. Efeito de diferentes substratos sobre o desenvolvimento de mudas de Acacia sp. Revista Árvore 2006; 30(2): 207-214. http://dx.doi.org/10.1590/ S0100-67622006000200007

Embrapa. Manual técnico da bracatinga (Mimosa scabrella Benth). Colombo: Embrapa-CNPF; 1988. 70 p.

Faustino R, Kato MT, Florêncio L, Gavazza S. Lodo de esgoto como substrato na produção de Senna siamea Lam. Revista Brasileira de Engenharia Agrícola e Ambiental 2005; 9(Suplemento): 278-282.

Fermino MH. Métodos de análise para caracterização de física de substratos [tese]. Porto Alegre: Universidade Federal do Rio Grande do Sul; 2003.

Freitas TAS, Barroso DG, Carneiro JGA, Penchel RM, Lamônica KR, Ferreira DA. Desempenho radicular de mudas de eucalipto produzidas em diferentes recipientes e substratos. Revista Árvore 2005; 29(6): 853-861. http:// dx.doi.org/10.1590/S0100-67622005000600003

Gomes JM, Couto L, Leite HG, Xavier A, Garcia SLR. Parâmetros morfológicos na avaliação da qualidade de mudas de Eucalyptus grandis. Revista Árvore 2002; 26(6): 655-664. http://dx.doi.org/10.1590/ S0100-67622002000600002

Guerrini IA, Trigueiro RM. Atributos físicos e químicos de substratos compostos por biossólidos e casca de arroz carbonizada. Revista Brasileira de Ciência do Solo 2004; 28: 1069-1076. http://dx.doi.org/10.1590/ S0100-06832004000600016

Hartmann HT, Kester DE, Davies FT Jr, Geneve RL. Plant propagation: principles and practices. Boston: Prentice-Hall; 2011. 915 p.

Kämpf AN. Substrato. In: Kampf AN. Produção comercial de plantas ornamentais. Guaíba: Agrolivros; 2005. p. 45-72.
Kratz D, Wendling I. Produção de mudas de Eucalyptus dunnii em substratos renováveis. Floresta 2013; 43(1): 125-136.

Mendonça AVR, Carneiro JGA, Freitas TAS, Barroso DG. Características fisiológicas de mudas de Eucalyptus spp submetidas a estresse salino. Ciência Florestal 2010; 20(2): 255-267.

Morais SMJ, Ataide PRV, Garcia DC, Kurtz FC, Oliveira OS, Wazlawick LF. Uso do lodo de esgoto da Corsan - Santa Maria (RS), comparado com outros substratos orgânicos. Revista Sanare 1997; 6: 44-49.

Novaes AB. Avaliação morfofisiológica da qualidade de mudas de Pinus taeda L., produzidas em raiz nua e em diferentes tipos de recipientes [tese]. Curitiba: Engenharia Florestal, Universidade Federal do Paraná; 1998.

Oliveira Junior AO, Cairo PAR,Novaes AB.Características morfofisiológicas associadas à qualidade de mudas de Eucalyptus urophylla produzidas em diferentes substratos. Revista Árvore 2011; 35(6): 1173-1180.

Paludzyszyn Filho E, Santos PET, Ferreira CA. Eucaliptos indicados para plantio no Estado do Paraná. Colombo: Embrapa Florestas; 2006. 45 p.

Röber R, Schaller K. Pflanzenernährung im Gerbau. 3rd ed. Sttugart: Ulmer; 1985. 352 p.

Rodrigues LRF. Técnicas de cultivo hidropônico e de controle ambiental no manejo de pragas, doenças e nutrição vegetal em ambiente protegido. Jaboticabal: FUNEP; 2002.

Rotta E, Oliveira YMM. Área de distribuição natural da bracatinga (Mimosa scabrella). In: Anais do Seminário Sobre Atualidades e Perspectivas Florestais, 4.: Bracatinga uma alternativa para reflorestamento. Curitiba: Embrapa-URPFCS; 1981. p. 1-24.

Trigueiro RM, Guerrini IA. Uso de biossólido como substrato para produção de mudas de eucalipto. Scientia Forestalis 2003; 64: 150-162.

Valeri SV, Corradini L. Fertilização em viveiros para produção de mudas de Eucalyptus e Pinus. In: Gonçalves JLM, Benedetti V. Nutrição e Fertilização Florestal. Piracicaba: IPEF; 2000. p. 167-189.

Wendling I, Delgado ME. Produção de mudas de araucária em tubetes. Colombo: Embrapa Florestas; 2008. 8 p.

Wendling I, Dutra LF. Produção de mudas de eucalipto por sementes. In: Wendling I, Dutra LF. Produção de mudas de eucalipto. Colombo: Embrapa Florestas; 2010. p. 13-47. PMid:22704086 PMCid:PMC3365363.

Wendling I, Gatto A. Substratos, adubação e irrigação na produção de mudas. Viçosa: Aprenda Fácil; 2002, 146 p.

Wendling I, Guastala D, Dedecek R. Características físicas e químicas de substratos para produção de mudas de Ilex paraguariensis St. Hil. Revista Árvore 2007; 31: 209-220. http://dx.doi.org/10.1590/ S0100-67622007000200003 